\title{
A Semantic-Aware Approach for Automatic Cloud Services Composition
}

\author{
${ }^{1 *}$ Hasan A. H. Naji, Chao Zhong $\mathrm{Wu}^{2}$ and Shu $\mathrm{Gao}^{3}$ \\ ${ }^{1 *, 2}$ Intelligent Transport Systems Research Center, Wuhan University of \\ Technology, 1040 Heping Avenue, Wuchang District, Wuhan, 430063, P.R. China. \\ ${ }^{3}$ School of Computer Science and Technology, Wuhan University of Technology, \\ 1040 Heping Avenue, Wuchang District, Wuhan, 430063, P.R. China. \\ 1*E-mail: hasanye1985@gmail.com, ${ }^{2}$ wucz@whut.edu.cn, ${ }^{3}$ gshu418@163.com
}

\begin{abstract}
Service composition is considered as a promising approach to generate composite services for satisfying services Consumers' needs using their preferences. Literature review shows that previous studies ignored issues that affect Service composition such as increasing composition time and no ranking for produced composite services. To address these issues, we propose a semantic-aware approach for automatic cloud services composition. Firstly, a semantic network of service is built using an efficient input-output matching algorithm. Secondly, a Forward-Chaining based algorithm is adopted to create composite services, according to the request of the Cloud Service Consumer. Thirdly, a hybrid ranking method based on similarity quality and QoS Criteria is implemented to select "the most suitable" composite service. The results of experiments provide evidence that our approach can effectively produce composite services for service consumer's request, and show benefits of consuming less composition time comparing to similar composition algorithms.
\end{abstract}

Keywords: Semantics, Cloud Service, Composition, Semantic Network, ForwardChaining, QoS

\section{Introduction}

Services Composition concentrates on defining and designing models and methods for performing composition on existing services to generate a more complex service, which satisfies the required functionalities of services consumers.

The composition work can be categorized into several types based on the approaches and technique used [1]; namely Static and Dynamic Composition, Model Driven Service Composition, Declarative Service Composition, Semantic Based Composition and Context Based Web Service Discovery and Composition.

For supporting automatic concepts in Services Composition, Semantic Annotation is presented. Ontology concepts are adopted to provide semantics to services. Semantic Services Composition [2] is a model that improves the flexibility of services in an application. An automatic services composer should integrate the required services, which are included in a composition plan, according to the user's specifications. The ability of automatically composing services and generating new composite services is main points for Semantic Services Composition. Furthermore, composite services [3] are dynamic, i.e., their components are able to be automatically selected at run-time.

There is a number of studies and researches conducted in the field of semantic and automatic cloud services composition. In [4], authors presented a framework called DynamiCoS for performing automatic services composition. Cloud services with semantics are used in DynamiCoS to support semantic reasoning which in turn facilitates automatic service discovery, selection and composition. Serrano Martín et al. [5] 
introduced a semantic-based approach of services provision for Cloud Service Composition. Domain ontological modelling and semantic links method are adopted for supporting services composition. Tan $\mathrm{W}$ et al. [6] proposed an approach for services composition. In the proposed approach, the inputs-output parameters of the service operations are modeled as colored places, and service operations themselves are represented as transitions along with input/output places. Composition plans are generated by linking services using input-output similarity matching. Peter Bartalos and Mária Bielikova [7] defined a QoS and behavior-aware approach for cloud service composition that deals with changes in the services and continuing arrival of new comings of composition queries. Omer A M and Schill A[8] introduced an input/output dependency based approach for automatic composition plans creation. In this approach, Input-Output dependency is described as directed graph and composition plans are created based on a graph traversal method called the topological sorting.

Most of the composition works ignored issues affect Service composition such as increasing composition time and no ranking for generated composite services. The main contribution of this paper is proposing and presenting in detail a semantic-aware approach for automatic cloud services composition that can explicitly consider the above issues. This approach can automatically find the most suitable composite service for satisfying a given request, which includes requested inputs, requested outputs and QoS weights, in a possible execution time. In addition, the approach provides a ranking method for the case wherein many composite services may produce from the composition process.

The rest of the paper is organized as follows: section 2 presents a Literature Review related to the study topic. Section 3 introduces the proposed approach in detail. Section 4 introduces experiments and analysis of our approach. In section 5, we summarize the content and the findings of this study.

\section{Literature Review}

\subsection{Cloud Services}

Fast development in the using of Cloud Computing technology leads to deploying more and more Cloud Services to cloud services pool. The services provided by Cloud providers are divided into following three main categories. Software as a Service (SaaS), Platform as a Service (PaaS) and Infrastructure as a Service (IaaS). Software as a Service (SaaS)[9]. This cloud service presents an application as a service without need to install and run any application on the computer of the cloud consumer. Consumers of SaaS Services, who are usually end users of applications or software administrators, can access this type of software using web browsers or mobile apps. Platform as a Service (PaaS). This type provides a developing platform as a service. In common, a PaaS service provides a complete development platform for organizations requiring a development instance of an application. Developers, testers, deplorers and application administrators are PaaS service consumers. Google App Engine is an example of a popular PaaS. Infrastructure as a Service (IaaS). This type of services provides infrastructure as a service, including server CPU cycles, data center space, storage resources, and database capacity. Services in IaaS are billed on a per use basis, the capacity can be increased in small increments, and the service is governed by stringent SLAs [10]. Typical IaaS consumers consist of system developers, network engineers, system administrators, monitoring engineers and IT managers.

This paper focuses on software as a service (SaaS) and presents study to discuss Cloud Service Composition. 


\subsection{Unified Ontology}

In this study, a unified ontology, which collects knowledge about cloud services, including Services Domains, Cloud service Characteristics and QoS Criteria, is built to unify the description of Cloud Services as described in [11]. This ontology defines a unified model that serves as a semantic-based repository, and plays a vital role in Cloud Service Advertisement, Selection and Composition. The Unified Ontology, which adopts OWL-S language [12], consists of classes and properties. The classes present the main terms and concepts of services, while properties present the relationships between the classes. The Unified Ontology contains the following classes as shown in Figure 1.

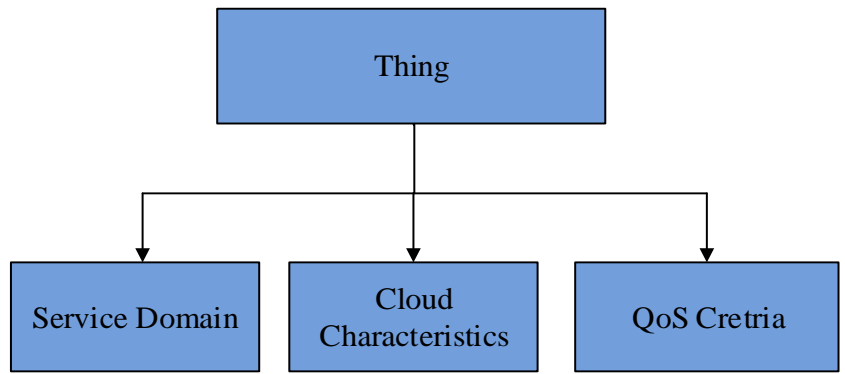

Figure 1. Snapshot of Main Concepts in Unified Ontology

The Unified Ontology contains main classes, including Service Domain, Cloud Characteristics and QoS Criteria.

\subsubsection{Service Domain}

A Service Domain presents classes for describing the functions of services according to their domain. Due to the complexity of covering and representing the whole domains of services, in this section we introduce the Finance domain as an example of Cloud Services domain.

Finance Domain provides semantic classes for Cloud Service descriptions in Finance area. A Finance Domain ontology is built to assist in Cloud Service advertisements. Figure 2 shows the main classes in the Finance Domain which has several key concepts including Currency, financial_Instrument, finance_operation, order, agent, quality and finacial_mean.

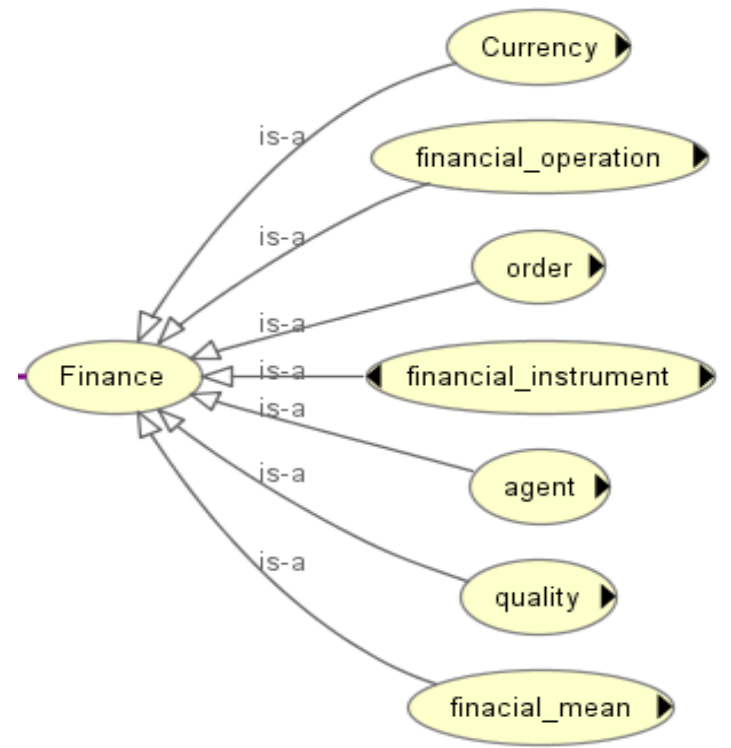

Figure 2. Snapshot of Main Classes in Finance Domain Ontology 
Each class represents its area using a subclass, for instance, Currency_Service and Conversion_Type are subclasses of Currency class.

\subsubsection{Cloud Service Characteristics}

Cloud Service Characteristics [13] are specific types of characteristics a Cloud Service provides to Cloud Services' Consumers. There are many main Cloud Service characteristic included in the Unified Ontology. The value partitions pattern is adopted in order to restrict the range of possible values for each characteristic into an exhaustive list, e.g. the range of possible values for the PaymentType characteristic is Free, Dynamic or PayOnDemand. A part of the ontology of Cloud service characteristics is presented as shown in Figure 3. There are several key classes in Cloud Service Characteristics' Ontology, including CloudServiceOpenness, PaymentType, LicenceClass, IntendedUserGroup and ServiceAgreement.

The main difference between the traditional services and Cloud Services is the latter are billed based on dynamic use. Rather than paying a fixed monthly or yearly charge, the customer only pays for the resources he uses on the consuming time.

\section{1) QoS Criteria}

QoS Criteria [14] of a Cloud Service are represented as concepts in the Unified Ontology. The QoS Criteria included in the QoS criteria ontology are Price, ResponseTime, Reputation, Reliability and Availability. The value of each QoS criteria guaranteed by the Cloud Service Providers are described using data properties, including hasResponseTime, hasAvailability, hasPrice, hasReputation and hasReliability. Data properties range can be one of the supported data types e.g. integer, string, boolean, etc. For example, in order to specify the price of the most popular plan of the Cloud Service Salesforce is 90 . In the unified ontology we use the following data property assertion: hasPrice 90 on the Salesforce service.

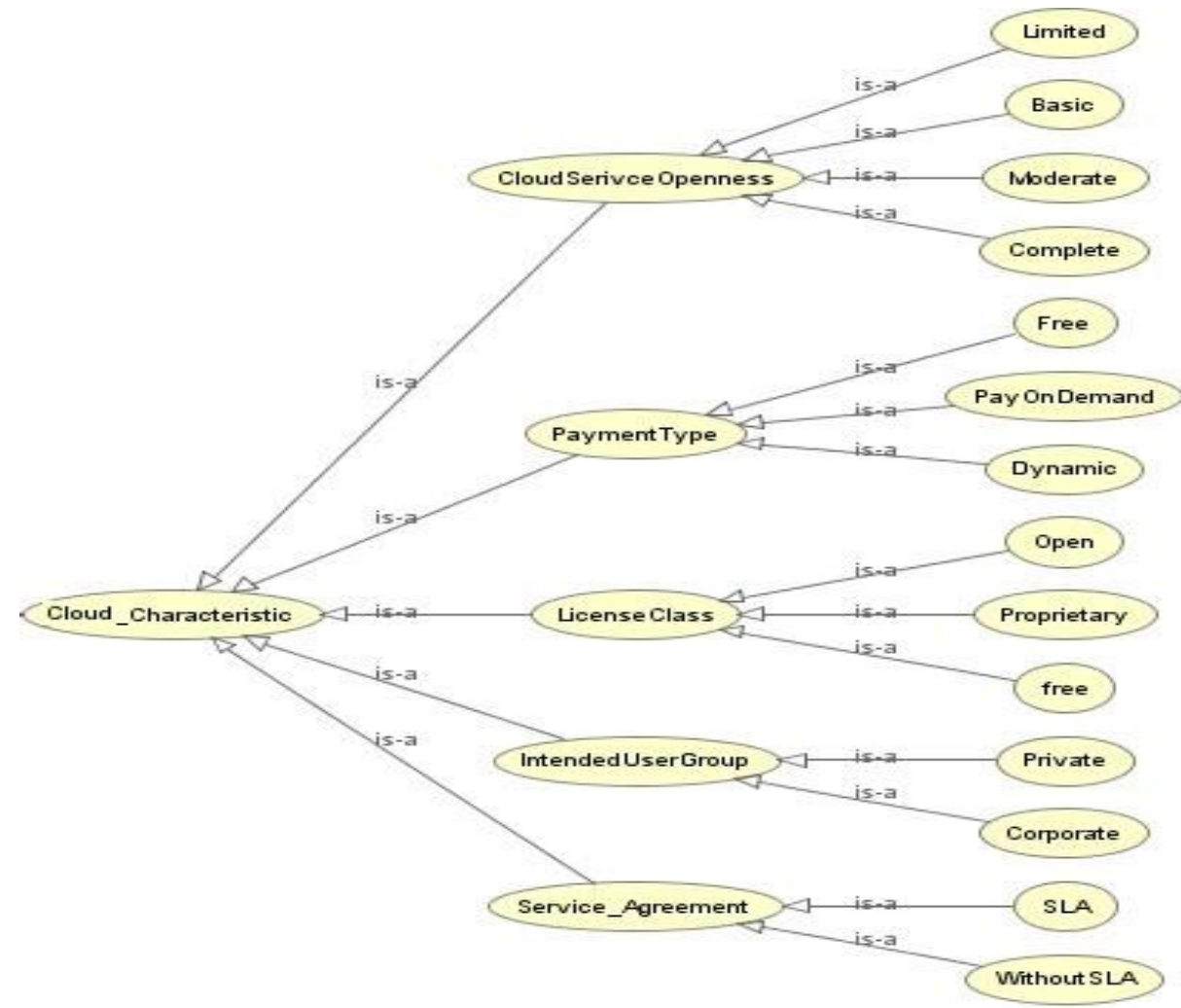

Figure 3. Snapshot of Main Concepts of Cloud Service Characteristics Ontology 


\section{Semantic-Aware Approach for Automatic Cloud Services Composition}

In this section, a Semantic-aware approach for automatic Cloud Services Composition is introduced. The Semantic-aware approach contains several novel algorithms including Output-Input matching algorithm for building a semantic network, Forward-Chaining algorithm for generating composite services and hybrid ranking method for finding the optimal composite service.

\subsection{Efficient Output-Input Matching Algorithm for Building a Semantic Network}

As the description of cloud services (i.e inputs-outputs, QoS Criteria and Cloud service characteristics) are stored in the Unified Ontology, the concept of Semantic Network [15] is adopted to facilitate services composition and to reduce consuming time for composition. A Semantic Network Sem $_{n e t}$ is a directed graph of cloud services (in a specific domain) where these services are linked by finding the matching similarity between their inputs and outputs. The matching similarity is performed using Semantic Casual Links [16]. Semantic Causal Link is a link of a semantic relationship between inputs and outputs of two cloud services.

The outputs and inputs of cloud services are mapped to concepts in the Unified Ontology. Therefore, retrieving the semantic relationship between two cloud services $s_{1}$ and $s_{2}$ is similar to discovering the semantic similarity between two semantic concepts $\left(\right.$ Out- $s_{1}$ and $\left.I n-s_{2}\right)$, where Out- $s_{1}$ is the concept of the output of cloud service $s_{l}$ whereas $I n-S_{2}$ is the concept of the output of cloud service $s_{2}$.

The function $\operatorname{Sim}\left(\mathrm{Out}-\mathrm{S}_{1}, \mathrm{In}-\mathrm{s}_{2}\right)$, called similarity function, is used to find semantic relationship between two cloud services $s_{1}$ and $s_{2}$. In other words, $\operatorname{Sim}\left(\right.$ Out $\left.-s_{1}, I n-s_{2}\right)$ function determines the semantic matching type between two concepts (Out-s $s_{1}$ and $\left.I n-s_{2}\right)$. The matching types [17] may obtained from $\operatorname{Sim}\left(\mathrm{Out}_{-} s_{1}, \mathrm{In}-s_{2}\right)$ are: (i) Exact (三): In this type, the output concept $O u t-s_{1}$ of a cloud service $s_{1}$ and the input concept $I n-s_{2}$ of a cloud service $s_{2}$ are semantically equivalent; formally: $O u t-s_{1} \equiv I n-s_{2}$. Exact type takes the value 1. (ii) Plug-In ( $\hat{O}$ ): In plug-in type, the output concept $O u t-s_{1}$ of a cloud service $s_{1}$ is a

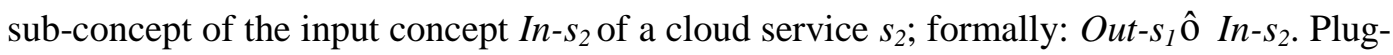
in type takes the value 1/2. (iii) Subsumed (o ): Subsumed type indicates that the output concept $O u t-s_{1}$ of a cloud service $s_{1}$ is a super-concept of the input $I n-s_{2}$ of a cloud service $s_{2}$; formally: Out- $s_{1}$ o $I n-s_{2}$. Subsumed type takes the value 1/3. (iv) Disjointed ( $\perp$ ): In this type, the output concept $O u t-s_{1}$ of a cloud service $s_{1}$ and the input $I n-s_{2}$ of a cloud service $s_{2}$ are none of the matching types mentioned above; formally: Out- $s_{1} \perp I n-s_{2}$. Disjointed type takes the value 0. For simplicity, the function $\operatorname{Sim}\left(\right.$ Out- $\left.s_{1}, I n-s_{2}\right)$ feeds back the corresponding value of matching type, such as 1 for Exact type.

As in [16], a Semantic Causal Link involves the semantic relationship between input and output of two cloud services. A Semantic Causal Link is defined as a triple $<s_{1}$, $\operatorname{Sim}\left(\right.$ Out- $\left.s_{1}, I n-s_{2}\right), s_{2}>. s_{1}$ and $s_{2}$ refer to two cloud services in a specific domain. Out- $s_{1}$ is a concept for an output of the cloud service $s_{1}$ whereas $I n-s_{2}$ is a concept for an input of the cloud service $s_{2}$. The function $\operatorname{Sim}\left(O u t-s_{1}, I n-s_{2}\right)$ feeds back the matching type (the corresponding value) according to the matching degree between the concepts Out- $s_{1}$, In- $s_{2}$ in the Unified Ontology. A Semantic Causal Link $<s_{1}$, $\operatorname{Sim}\left(\right.$ Out- $\left.s_{1}, I n-s_{2}\right), s_{2}>$ represents that: (i) $s_{1}$ precedes $s_{2}$, since an output of $s_{1}$ is consumed by an input of $s_{2}$ and (ii) No intermediate cloud service is existed between $s_{1}$ and $s_{2}$.

A Valid Causal Link is a Semantic Causal Link $<s_{1}, \operatorname{Sim}\left(O u t-s_{1}, I n-s_{2}\right), s_{2}>$ when the

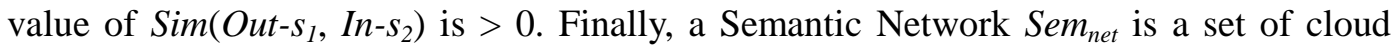
services (in a specific domain) where these services are linked by Valid Casual Links.

For instance: we have a set of cloud services in the domain of weather. Each cloud service is represented as the tuple $s_{i}=\langle i d$, name, ins, outs $>$ where id indicates a cloud service identifier, name denotes a cloud service name, ins is a set of input(s) of the cloud 
service $s_{i}$ and outs is a set the output(s) of the cloud service $s_{i}$. For the sake of simplicity, we assume that each cloud service has one input and one output. Table 1 shows of cloud services and their descriptions.

$\operatorname{Sim}\left(\right.$ Out $\left.-s_{1}, I n-s_{2}\right)$ function is used to determine the semantic matching type between inputs and outputs in Table 1. Figure 4 shows a part of the semantic network, which is built according to the descriptions of cloud services in Table 1.

Table 1. Cloud Services' Descriptions

\begin{tabular}{|c|c|c|c|c|}
\hline & Id & Name & Input & Output \\
\hline $\mathrm{s}_{1}$ & 1 & FindZipCode & City & Zipcode \\
\hline $\mathrm{s}_{2}$ & 2 & FindLatLong & Zipcode & Lat/long \\
\hline $\mathrm{s}_{3}$ & 3 & GetPressure & Lat/long & Press \\
\hline $\mathrm{s}_{4}$ & 4 & GetWeather & Lat/long & weather \\
\hline $\mathrm{s}_{5}$ & 5 & Findweather & City & weather \\
\hline $\mathrm{s}_{6}$ & 6 & Findclothesforweather & Weather & clothes \\
\hline
\end{tabular}

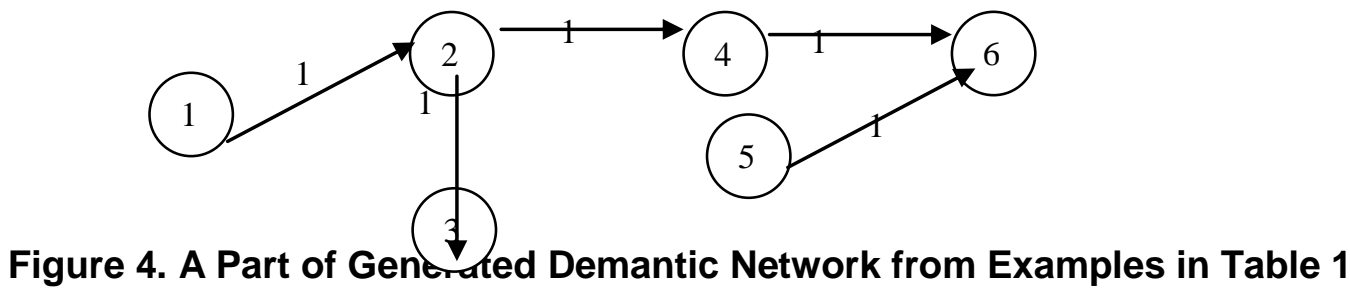

The output of the service $s_{1}$ is similar (exact) to the input of the service $s_{2}$; and the output of the service $s_{2}$ is similar (exact) to the input of the services $s_{3}$ and $s_{4}$. Algorithm 1 illustrates the process of matching cloud services and building a Semantic Network.

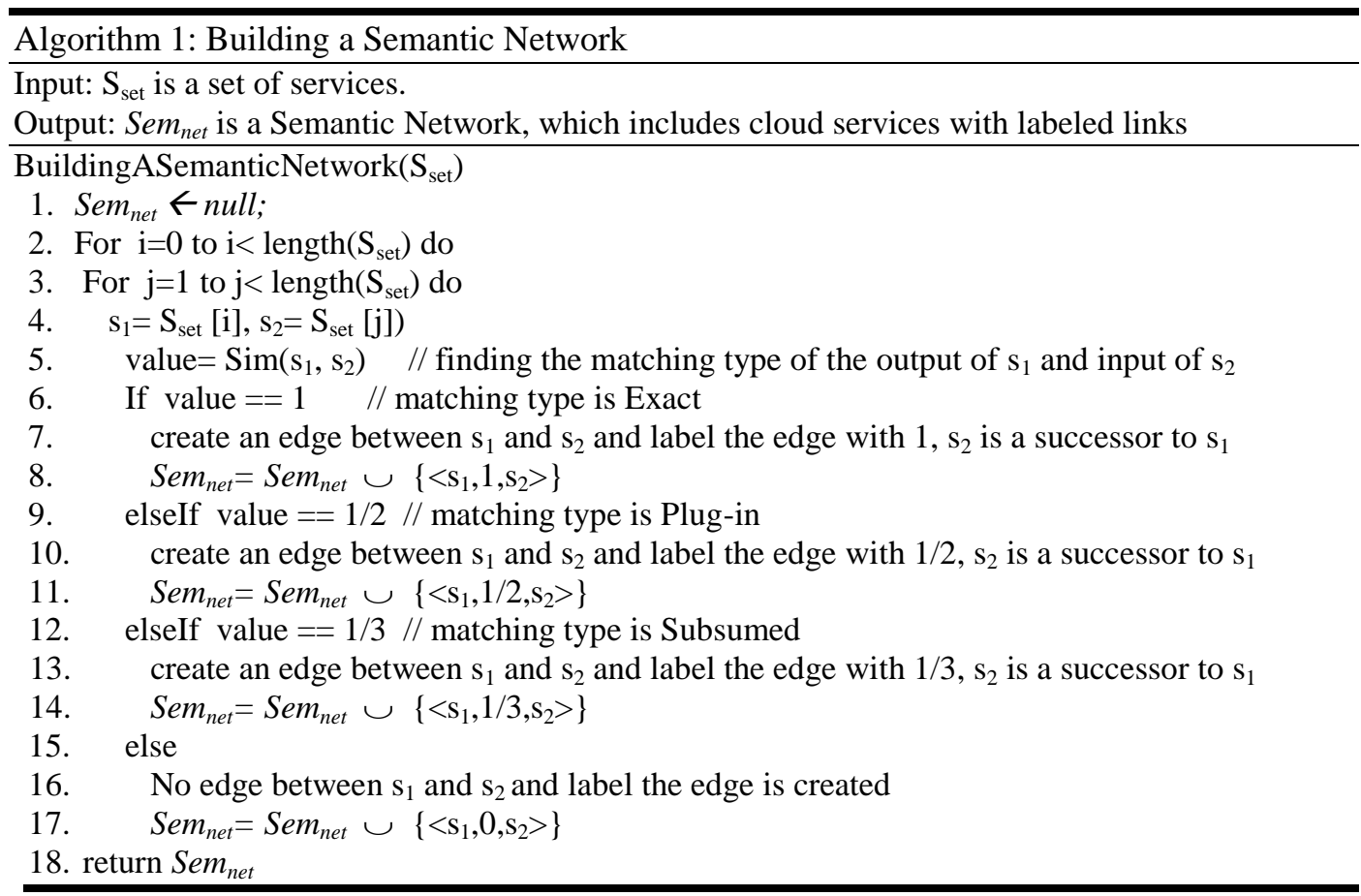

In Algorithm 1, $\operatorname{Sim}\left(\mathrm{s}_{1}, \mathrm{~s}_{2}\right)$ provides a value that represents the matching type between services. The result of the algorithm is a set of linked services that form a Semantic Network. 


\subsection{Forward-Chaining based Algorithm for Finding Composite Services}

In order to automatically find a composite service that satisfies the request of a Cloud Service Consumer, a novel algorithm is proposed. The proposed algorithm adopts Forward-Chaining and Depth-First methods[18] to generate composite services. The algorithm takes the input of the request as the start point, and the output of the request as the end point, and then finds all composite services may generate between these points.

A composite service[18] is a set of linked services, in a Semantic Network, that forms a path, which begins with the requested input and ends with the requested output.

Assume there is a semantic network $\operatorname{Sem}_{n e t}$ and a request $R=\langle$ ins,outs $\rangle$, where ins is a set of requested input(s) and outs is a set of requested outputs. A set of composite service $C S_{\text {set }}=\left\{\right.$ path $_{1}, \ldots$, path $_{u}, \ldots$, path $\left._{o}\right\}$ where path $_{u}$ is a composite service which is automatically generated as solutions for the request using Depth-First and Forward-Chaining methods. Each path, which presents a composite service, begins with the requested input and ends with the requested output.

Algorithm 2 shows the main steps of generating composite services by finding paths using Forward-Chaining method.

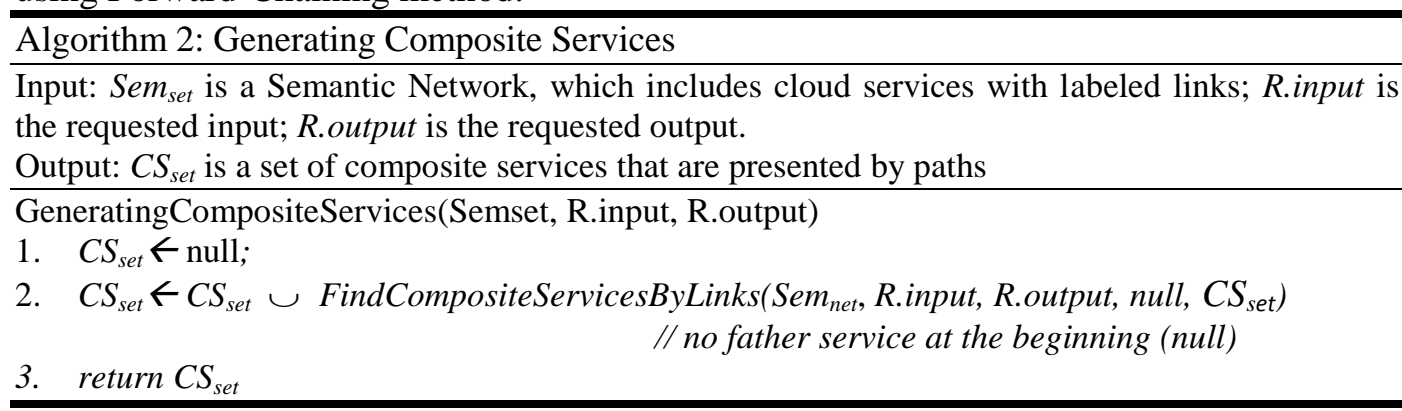

Algorithm 2 generates a set of composite services $C S_{\text {set }}$. A function of finding all possible composite service, called FindCompositeServicesByLinks, is invoked with several inputs. These inputs are as follows: $\operatorname{Sem}_{n e t}$ is a Semantic Network, which includes cloud services with labeled links; R.input is the requested input whereas R.output is the requested output. father is the up service of the current services, the initial value of father is null. $C S_{\text {set }}$ is an intermediate set of composite services that will be updated by adding new composite services when FindCompositeServicesByLinks function is invoked. $C S_{\text {set }}$ will be fed back as the final output of invoking FindCompositeServicesByLinks function.

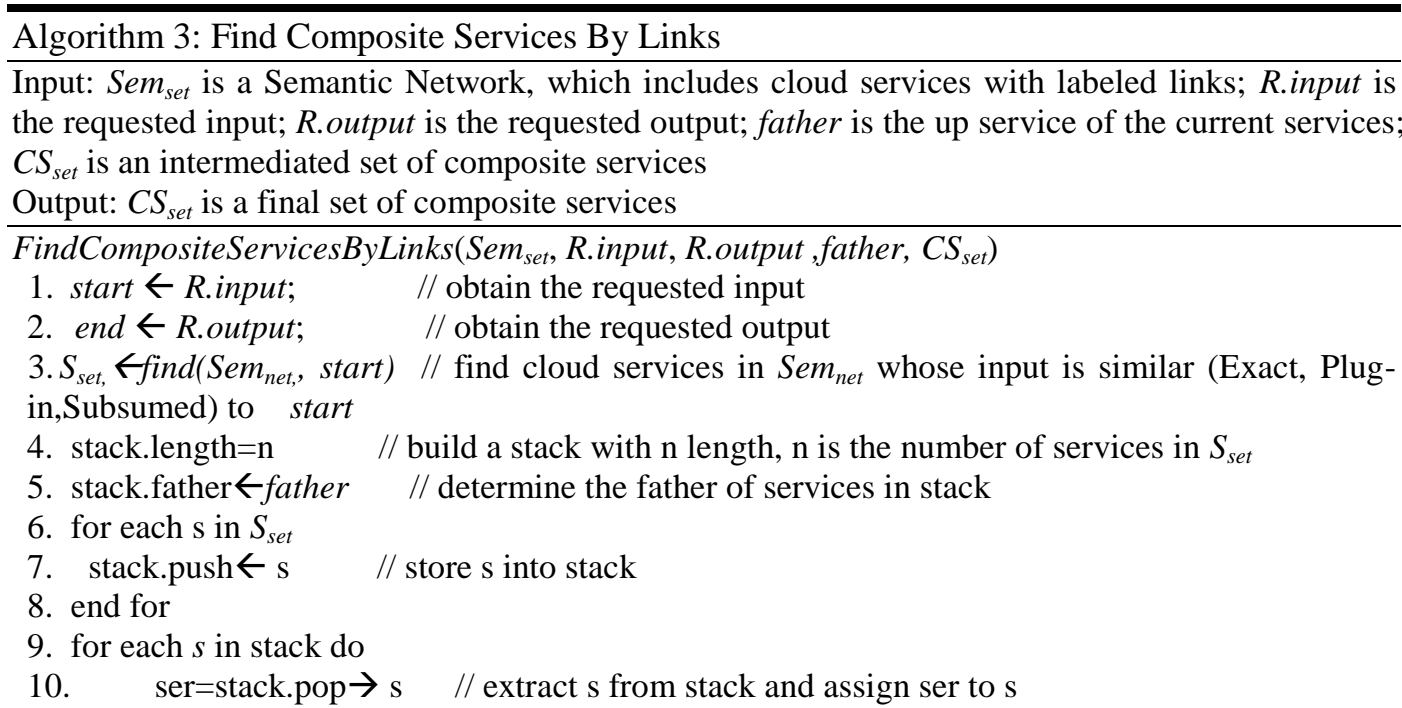


11. if ser.output ! = end // if ser.output is not similar to end

12. father $\leftarrow$ ser

13. FindCompositeServiceByLinks $\left(\operatorname{Sem}_{n e t}\right.$, ser.output, end, father, $\left.\mathrm{CS}_{\mathrm{set}}\right)$ // recursion

14. else

15. $\quad$ links $\leftarrow$ null;

16. $\quad$ links $\leftarrow$ GetLinksFromEndToStart (ser, R.input, R.output, links) // find links from start to end

17. $\quad$ path $\leftarrow$ generatePath(links) // generate a path using links

18. $C S_{\text {set }} \leftarrow C S_{\text {set }} \mathrm{U}$ path

19. end if

20. end for

Algorithm 3 finds the possible composite services in the Semantic Network Sem net $_{\text {. }}$ Firstly, R.input and R.output are assigned to start and end variables, respectively. Secondly, the services whose input is similar (Exact, Plug-in or Subsumed) to start are stored in the set $S_{\text {set }}$. Thirdly, a stack is built and its length is assigned to $n$ (number of services in $S_{\text {set }}$ ). The father of this stack is assigned to father value and the services in $S_{\text {set }}$ are stored in stack. Fourthly, all services in stack are extracted one by one. For each extracted service $s, s$ is assigned to ser, if the output of ser is not similar to end, then we recursively call the function FindCompositeServicesByLinks, otherwise, the function GetLinksFromEndToStart is invoked to find the links from R.output (end) to R.input (start). A path, which begins start to end from R.input (start), is generated by ordering the links to by invoking generatePath. Finally, path is added to $C S_{\text {set }}$.

Algorithm 4: Get Links from End to Start

Input: ser is the current service; R.input is the requested input; R.output is the requested output; links is an intermediated set of links

Output: links is the final set of links from End to Start

GetLinksFromEndToStart(ser, R.input,R.output,links)

1. father $\leftarrow$ Findfather(ser) // find the father of service ser

2. if father is not null and ser.output is not similar to R.output // the current service is in the middle and the output of ser is not similar (Exact, Plug-in or Subsumed) to R.input

3. $\quad$ links $\leftarrow$ links $\cup<$ father.output,ser.input $>$

4. $\quad$ ser $\leftarrow$ father

5. links $\leftarrow$ links $\cup$ GetLinksFromEndToStart (ser, R.input, R.output, links) // recursion

6. elseif father is not null and ser.output is similar to R.output // the current service is at the bottom

ser is similar

near to R.output and the output of

R.input

7. $\quad$ links $\leftarrow$ links $\cup<$ ser.output, R.output $>$

8. $\quad$ links $\leftarrow$ links $\cup<$ father.output, ser.intput $>$

9. $\quad$ ser $\leftarrow$ father

10. links $\leftarrow$ links $\cup$ GetLinksFromEndToStart (ser, R.input, R.output, links) // recursion

11. elseif father is null and ser.input is R.input // the current service is at the top near to R.input and the

Subsumed) to R.input

output of ser is similar (Exact, Plug-in or

12. links $\leftarrow$ links $\cup<R$.input, ser.input $>$

13. end if

Algorithm 4 finds the links from R.output to R.input. Firstly, it finds the father service of the current service ser. Secondly, the position of the current service ser is checked. If ser is in the middle (ser is not the first service or the last service), the link between ser 
and father and then ser is assigned to father and the algorithm invokes itself again. If ser is in the bottom (ser is the last service), the link from ser to R.output and the link from father to ser are obtained and then ser is assigned to father and the algorithm invokes itself again. If ser is in the top (ser is the first service), the link between R.input and ser is obtained. Finally, the whole links from R.output to R.input are returned as the output of this algorithm. The flow chart of the forward-Chaining algorithm is shown in Figure 5.

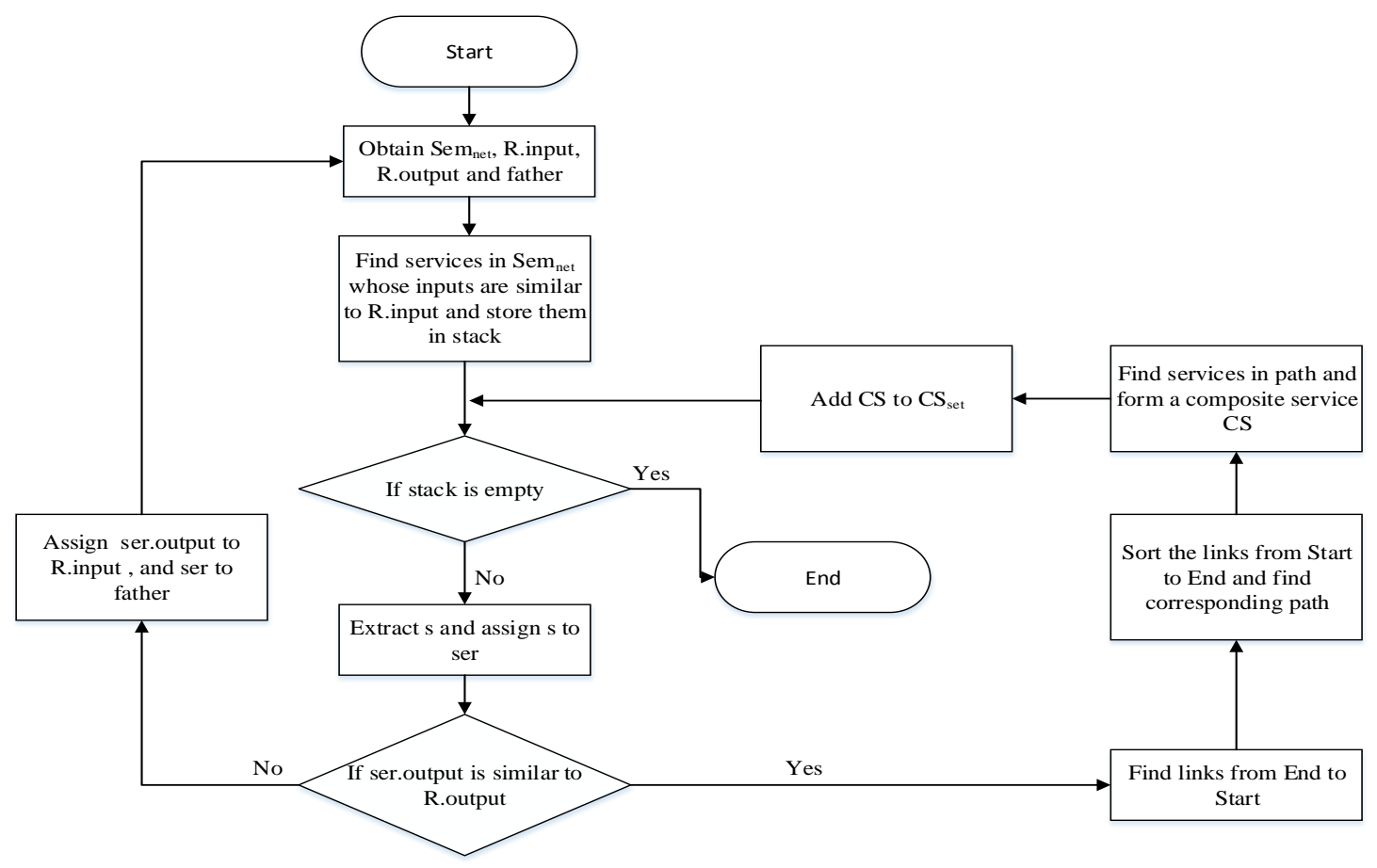

Figure 5. Flow Chart in Forward-Chaining Algorithm

\subsection{Hybrid Ranking Method for Selecting Optimal Composite Service}

Forward-Chaining based algorithm produces a list of composite services in which each composite service can satisfy the functional requirement of the cloud services consumer. Therefore, a ranking method is required to find the optimal composite service. To this ends, a hybrid ranking method is adopted. This method goes in two steps, Similarity Quality Ranking and QoS-Aware Ranking.

\subsubsection{Similarity Quality Ranking}

In this step, the Similarity Quality of the each composite service is computed by multiplying the match type values of the links of the path which forms the composite service. The composite service that its path has the highest value is sent back to the service consumer as the optimal composite service. The Similarity Quality of a composite service $C S_{u}$, which is formed by path $_{u}$, is computed as follows.

$$
\operatorname{Sim}_{\text {quality }}\left(\text { path }_{u}\right)=\operatorname{Sim}\left(\operatorname{start}, s_{j} . \text { input }\right) \times \operatorname{Sim}\left(s_{j+1} . \text { output }, s_{j+2} . \text { input }\right) \times \ldots \times \operatorname{Sim}\left(s_{j+m-1} \text {.output }, \text { end }\right)
$$

where $\operatorname{Sim}$ is the similarity function for obtaining the value matching type of two concepts(the value is $1,1 / 2$ or $1 / 3$ ) as in section 3.1, start and end indicate the concepts of requested input and output of the request respectively. The parameters in Sim function are the links that form the $\mathrm{path}_{u}$ of the composite service $C S_{u}$. 


\subsubsection{QoS-aware Ranking}

This step is used in the case that the highest value of Similarity Quality is obtained and found by more than one composite service. Thus, QoS-aware ranking [19] is used to find the most suitable composite service, which has the highest Utility Value.

Assume that the Forward-Chaining Algorithm produces a set of composite services $C S_{\text {set }}=\left\{\right.$ path $_{1}, \ldots$, path $_{w}, \ldots$, path $\left._{o}\right\}$ and each of these paths has the same highest Similarity Quality value. Then, Utility Value is required to rank and find the most suitable path (composite service) in respect of QoS Criteria. As each path $_{u}$ forms a corresponding composite service $C S_{u}$ and for the sake for simplicity, in the following we use the concept composite service $C S_{u}$ instead of path $_{u}$.

A composite service $C S_{u}$, contains $n$ cloud services, such that $C S_{u}=\left\{\mathrm{s}_{\mathrm{j}}, \mathrm{s}_{\mathrm{j}+1}, \ldots, \mathrm{s}_{\mathrm{j}+\mathrm{n}-1}\right\}$, and each service $s_{j}$ has five QoS Criteria $c_{k}$, namely Price, Duration, Availability, Reliability and Reputation as described in the Unified Ontology.

$C S_{u} \cdot \mathrm{c}_{\mathrm{k}}$ indicates the aggregated value [19] of QoS Criteria $c_{k}$ of a composite service $C S_{u} \cdot C S_{u} \cdot c_{\mathrm{k}}$ is computed as shown in Table 2.

Table 2. Aggregated Value of QoS Criteria in Sequential Model

\begin{tabular}{|c|c|c|c|c|c|}
\hline $\begin{array}{c}\text { QoS } \\
\text { Criteria }\end{array}$ & Price & Duration & Availability & Reliability & Reputation \\
\hline $\begin{array}{c}\text { Aggregated } \\
\text { Value }\end{array}$ & $\sum_{j=1}^{n} s_{j} \cdot c_{k}$ & $\sum_{j=1}^{n} s_{j} \cdot c_{k}$ & $\prod_{j=1}^{n} s_{j} \cdot c_{k}$ & $\prod_{j=1}^{n} s_{j} \cdot c_{k}$ & $\frac{1}{n} \sum_{j=1}^{n} s_{j} \cdot c_{k}$ \\
\hline
\end{tabular}

where $\mathrm{s}_{\mathrm{j}}$ is a component service in the composite service $C S_{u}$ and $\mathrm{s}_{\mathrm{j}} . \mathrm{c}_{\mathrm{k}}$ indicates QoS criteria value of the $s_{j}$.

The utility value [20], which is denoted by $U C S_{u}$, of a composite service $C S_{u}$ is computed as follows:

$$
U C S_{u}=\sum_{k+} w_{k+} \times \frac{M A X_{k+}-C S_{u} \cdot c_{k+}}{M A X_{k+}-M I N_{k+}}+\sum_{k-} w_{k-} \times \frac{C S_{u} \cdot c_{k-}-M I N_{k-}}{M A X_{k-}-M I N_{k-}}
$$

where $k+$ indicates a positive QoS criteria such as availability and reputation whereas $k$ - denotes a negative QoS Criteria such as Price and Duration. $C S_{u} \cdot \mathrm{c}_{\mathrm{k}}$ denotes the aggregated $Q o S$ value of the composite service $C S_{u}, M A X_{k}$ and $M I N_{k}$ indicate the maximum and minimum value of QoS Criteria $k$ of available composite services.

Assume there is a set of composite services $C S_{\text {set }}=\left\{C S_{1}, \ldots, C S_{u}, \ldots, C S_{o}\right\}$, as each $C S_{u}$ is formed using a corresponding path path $_{u}$, then the maximum value of QoS criteria $k$ is computed as $\operatorname{MAX}_{k}=\underset{u=1}{\operatorname{Max}}\left(C S_{u} \cdot c_{k}\right)$, and the minimum value of QoS Criteria $k$ is computed as $\operatorname{MIN}_{k}=\underset{u=1}{\operatorname{Min}}\left(C S_{u} \cdot c_{k}\right)$.

After computing the Utility Value of all $C S_{u}$, which has similar highest value of the Similarity Quality, the composite service that provides the Maximum Utility Value will be fed back to Cloud Service Consumer as the most suitable composite service.

\section{Experiment and Analysis}

In this section, several experiments are conducted to validate our approach, which achieves better composition efficiency than traditional automatic composition methods, e.g., SAT [21], while finding the optimal composite service using a data set called OWLSTC [22]. In the following, the content of our experiment is introduced in detail. The content includes Experimental Setting and Experiment Result and Analysis. 


\subsection{Experiment Setting}

\subsubsection{Data}

The experiments of our work using OWL-TC test collection obtained from http://projects.semwebcentral.org/projects/owls-tc/. With modifying the data set content to be fit with data in the unified ontology, the data set provides around 5000 services and 100 queries (requests) specified with OWL-S in several domains.

All experiments have been performed on a PC using a $4 \mathrm{G}$ memory and i7 CPU. In addition, several programs and software are used such as operating system: Windows 8 , Web Server: Tomcat 7.0 and plug-ins: Axis 1.4, Pellet 4.0, Portege 3.1 and Jena 3.2.

4.1.2 Evaluation Method

For the aim of evaluating our experiments, we implemented a scenario, where a request includes a requested input and a requested output. The request of service consumer is solved using the following approaches.

(1) SAT Approach [21]: This approach presents services composition based on the semantic dependency between services using Input-Output matching in order to link these services and then using Casual Link Matrix to build composite services.

(2) Our Approach: This approach includes an Output-Input algorithm for building Semantic Network, Forward-Chaining algorithm, and Hybrid ranking method.

\subsection{Experiment Results and Analysis}

In this section, we compare and analyze the performance of composition using our approach and the approach in SAT in terms of execution time and Successful Rate.

4.2.1 Successful Rate

Successful Rate of composition is the rate of the ability of a specific approach to successfully finding the solution (composite service) of a given request. In order to evaluate the Successful Rate of our approach and SAT approach, the testing dataset was grouped into five groups as follows: 200, 300, 400, 500, 1000 and 100 requests were provided to find solution (composite service). Figure 6 shows the Successful Rate of the two approaches in respect with the five groups of testing dataset.

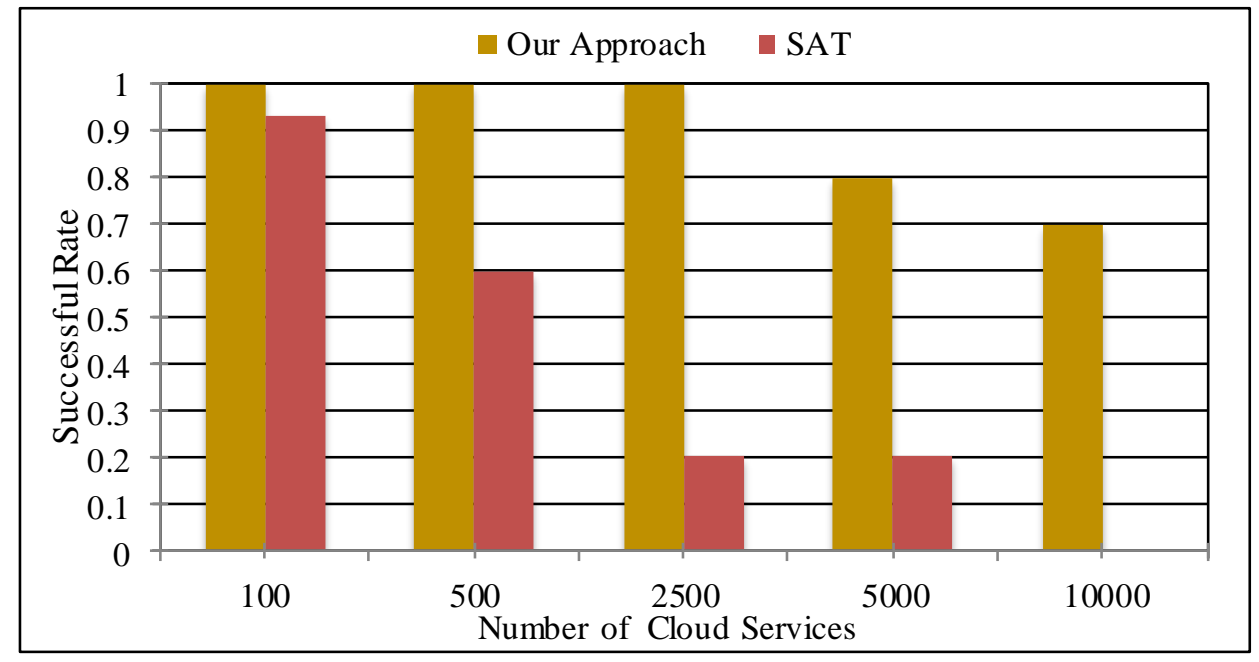

Figure 6. Comparison of the Successful Rate of the Two Approaches

Since a testing dataset included small number of services the two approaches find solutions, but since a testing dataset included big number of services SAT performs low Successful Rate and reached 0 since reached 10000 services. While our approach had a 
low effect since the number of services in a testing dataset went small, and this means that performs better than SAT and has a high ability to find solution for requests.

4.2.2 Execution Time

In this experiment, the execution time was studied using varied numbers of cloud services. The numbers of services were varied from 200 to 1000 . The execution time indicates the consuming time for achieving a request of the service consumer. Figure 7 shows the execution time in terms of varied numbers of cloud services.

As Figure 7 shows, SAT was faster as small number of services whereas our approach was faster as large number of cloud services. The execution time of both approaches increased as the number of cloud services increased. However, the execution time of our approach degraded more gracefully than in SAT as building a semantic network, in our approach, is an offline process thus the consuming time of building a semantic network is trivial and not considered. Figure 8 shows the execution time for SAT and our approach in the case that the number of services is fixed to 1000 and the number of requests is varied from 20 to 100. As Figure 8 shows, the gap in execution time between SAT and our approach is widened as the number of requests increased. This proves that our approach scales better than the SAT approach as the number of requests increases.

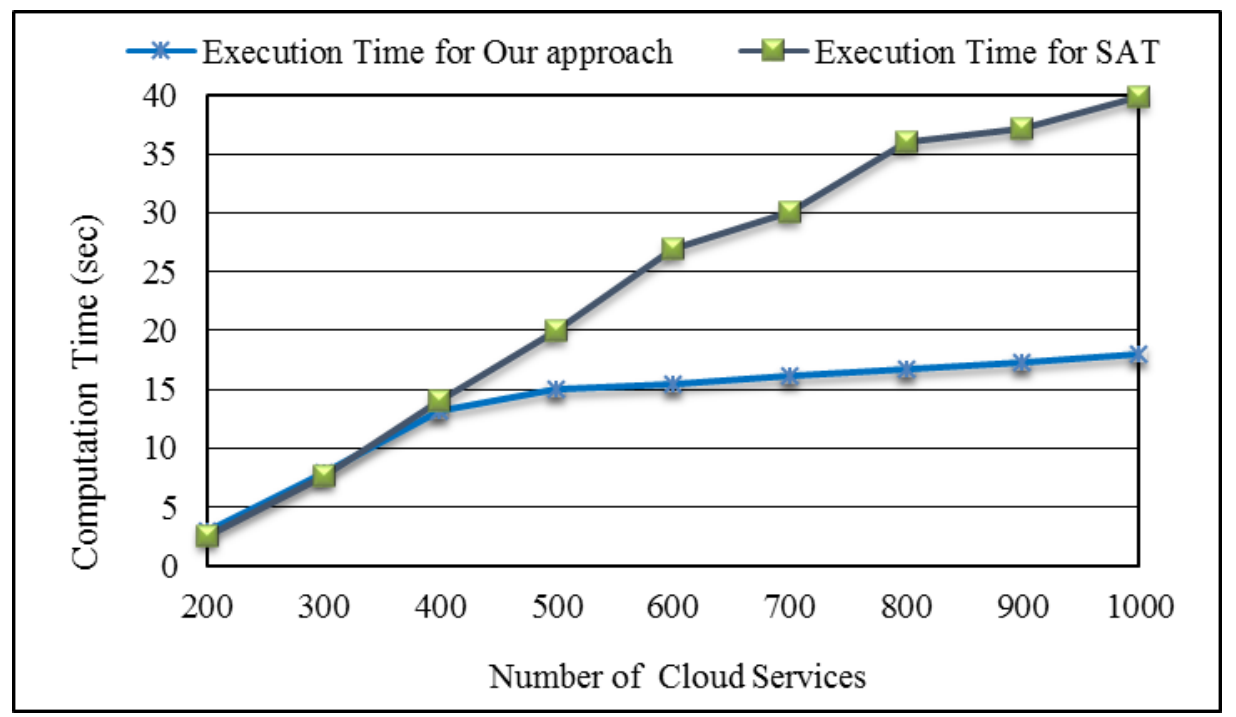

Figure 7. Execution Time Comparison in Term of Varied Number of Services

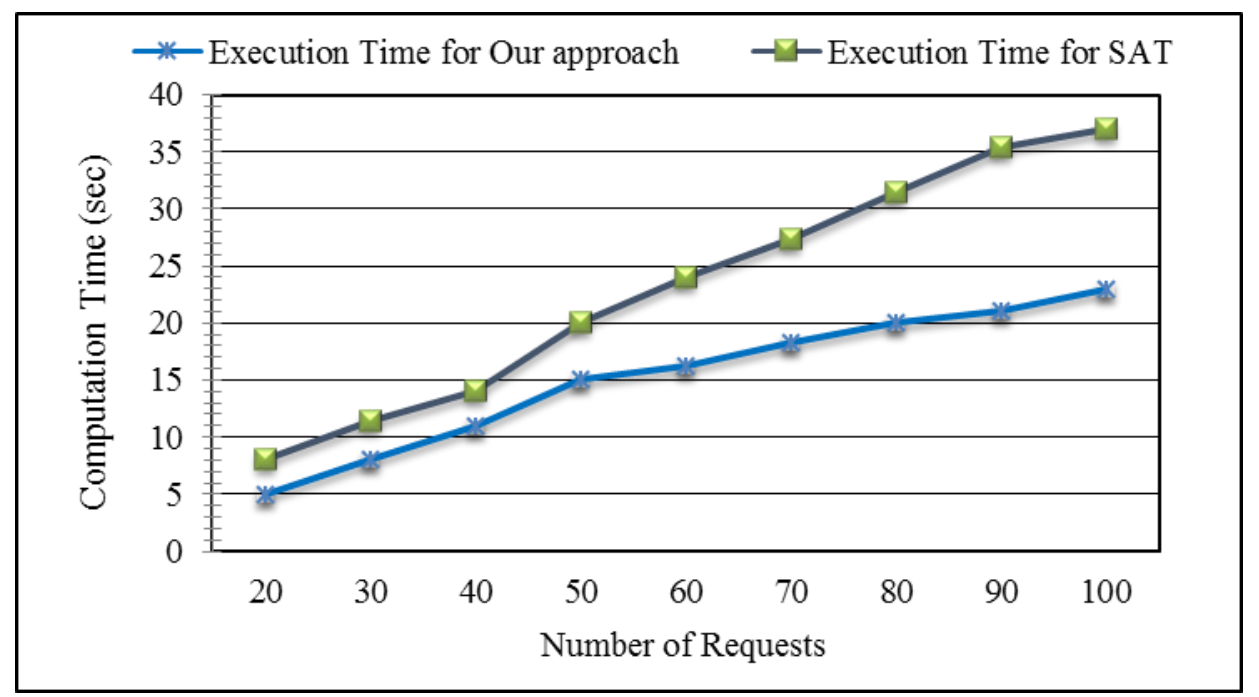

Figure 8. Execution Time Comparison between Our Approach and SAT 


\subsubsection{QoS Optimality}

Our approach adopted QoS-based Ranking method to select the optimal composite service according to the requirements of services consumer. This enhanced the successful rates and efficiency of composition as selecting the best composite service among a set of composite services that satisfies the functional requirement. SAT [21] ignores the important role can QoS Criteria play for ranking the available composite services.

\section{Conclusions}

The ability of Cloud Services to compose and integrate loosely coupled applications has attracted the attentions of researchers in the field of Automatic Cloud Services Composition. This paper proposed a semantic-aware approach for automatic Cloud Services Composition. The proposed approach contains three steps. Firstly, an interconnected network (called semantic network) is built using an efficient approach of the similarity (Outputs-Inputs similarity) among semantic concepts of cloud services. Secondly, a Forward-Chaining method is adopted to create composite services that functionally satisfy the Service Consumer's request (requested inputs and requested outputs). Thirdly, as several composite services may be generated from the ForwardChaining method, a hybrid ranking method based on similarity quality and QoS Criteria is adopted and used to select " the suitable" composite service for the Service Consumer.

In this papers, more attention was paid on both aspects: 1) building a semantic network, which is an offline process and then its consuming time is trivial, 2) performing ranking composite services process which selects the suitable composite service using services consumer's preferences.

It is clear from the above results that our approach significantly outperforms the traditional approach SAT on the consuming time of services composition and provides an efficient ranking method for generated composite. The study results clearly suggest that using our approach may be a better choice for automatic services composition.

Future work may involve services composition for other types of cloud services (such as IaaS) and may consider designing and implementing methods and models taking into account computing resources such as CPU cycles, data center space, storage resources.

\section{Acknowledgement}

This study is sponsored by the National Key Technology R\&D Program (2014BAG01B03), the National Natural Science Foundation of China (51105286, 61203236) and the Academic Leader Project of Wuhan (2012711304457) and the Fundamental Research Funds for the Central Universities (WUT: 2014-IV-137).

\section{References}

[1] Leitner, Philipp, Waldemar Hummer, Schahram Dustdar. "Cost-based optimization of service compositions", IEEE Transactions on Services Computing. Vol. 3, no. 2,(2013), pp. 239-251.

[2] Joshi K.P, Yesha Y, Finin T. "Automating Cloud Services Life Cycle through Semantic Technologies". IEEE Transactions on Services Computing”, vol.7, no.1,(2014) pp. 109-122.

[3] Quan Z Shenga, Xiaoqiang Qiao, Athanasios V Vasilakosc, Claudia Szaboa, Scott Bournea, Xiaofei Xu. "Web services composition: A decade's overview". Information Sciences. vol. 28, (2014), pp. 218-238.

[4] Eduardo Gonçalves da Silva, Luís Ferreira Pires,Marten van Sinderen.” Towards runtime discovery, selection and composition of semantic services". Computer communications. vol.34, no.2, (2011).pp. 159-168.

[5] Martín Serrano, Lei Shi, Mícheál Ó Foghlú, William Donnelly. "Cloud services composition support by using semantic annotation and linked data. Knowledge Discovery". Knowledge Engineering and Knowledge Management. (2013),pp. 278-293.

[6] Wei Tan, Yushun Fan, MengChu Zhou, Zhong Tian. "Data-driven service composition in enterprise SOA solutions: a Petri net approach". IEEE Transactions on Automation Science and Engineering. vol.7, no.3, (2010), pp. 686-694. 
[7] Bartalos, P, Bieliková M. "QoS aware semantic web service composition approach considering pre/postconditions". In Web Services (ICWS), 2010 IEEE International Conference on, (2010),pp.345352.

[8] Omer A M, Schill A. Dependency based automatic service composition using directed graph. Next Generation Web Services Practices, NWESP'09. Fifth International Conference on. IEEE, 2012, pp. 7681.

[9] Noor T.H, Sheng Q.Z, Ngu, A.H.H, Dustdar S.”Analysis of Web-Scale Cloud Services”. IEEE Internet Computing. vol.18, no.4,(2014), pp.55-61.

[10] Lars Nielsen. "The Little Book of Cloud Computing: Including Coverage of Big Data Tools". New Street Communications, LLC.(2013).

[11] Afify, Yasmine M..”Cloud Services Discovery and Selection: Survey and New Semantic-Based System". Bio-inspiring Cyber Security and Cloud Services: Trends and Innovations. Springer Berlin Heidelberg, (2014). pp.449-477.

[12] Bernardo Cuenca Grau., et al. "OWL 2 Web Ontology Language: Profiles (Second Edition)".http://www.w3.org/TR/owl2-profiles/.(2015.12.11).

[13] Afify, Yasmine M., et al. "Concept Recommendation System for Cloud Services Advertisement. Advanced Machine Learning Technologies and Applications". Springer International Publishing,(2014). pp.57-66.

[14] Zou G, Lu Q, Chen Y, et al. "QoS-aware dynamic composition of Web services using numerical temporal planning”. Services Computing, IEEE Transactions on. vol.7, no.1, (2014),pp. 18-31.

[15] Hasan Naji,A.H, Gao Shu, Al-Gabri Malek. "An optimal semantic network-based approach for web service composition with qos", Telkomnika. vol.11, no 8, , (2013),pp. 4505-4511.

[16] Cassar G, Barnaghi P, Wang W, et al. "A hybrid semantic matchmaker for IoT services". Green Computing and Communications (GreenCom), 2012 IEEE International Conference on. IEEE, (2012), pp. 210-216.

[17] Dong H, Hussain F K, Chang E. "Semantic Web Service matchmakers: state of the art and challenges. Concurrency and Computation: Practice and Experience". vol.25,no.7, (2013), pp. 961-988.

[18] Khakhkhar S, Kumar V, Chaudhary S. "Dynamic Service Composition". International Journal of Computer Science and Artificial Intelligence. vol. 2, no.3, (2012), pp. 32-42.

[19] Zheng, Zibin, et al. "QoS ranking prediction for cloud services". IEEE Transactions on Parallel and Distributed Systems, vol.24, no.6, (2012), pp. 1213-1222.

[20] M. Alrifai, T. Risse, W. Nejdl. "A hybrid approach for efficient web service composition with end-toend QoS constraints”. ACM Transactions on the Web. vol.6, no.2, p. 7. http://dx.doi.org/10.1145/2180861.2180864.

[21] Kil, Hyunyoung, Wonhong Nam. "SAT Solving Technique for Semantic Web Service Composition. Computer Applications for Web, Human Computer Interaction, Signal and Image Processing, and Pattern Recognition”. Springer Berlin Heidelberg. (2012), pp. 167-172.

[22] http://projects.semwebcentral.org/projects/owls-tc/, (2016.01.15).

\section{Authors}
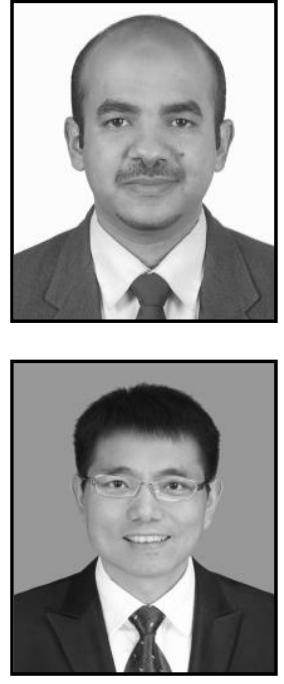

Hasan.A.H Naji, He is working as an Assistant Professor at Wuhan University of Technology, Wuhan, China. He has received her Master Degree and PhD in Computer science and Technology from Wuhan University of Technology, Wuhan, China. His areas of research are Service-Oriented Computing, Transportation data analysis, and Data mining.

Chao Zhong Wu, He is working as a Fulltime Professor at Wuhan University of Technology, Wuhan, China. He has received her Master Degree and $\mathrm{PhD}$ in Transportation Engineering from Wuhan University of Technology, Wuhan, China. His areas of research are Transportation data analysis, Driver behavior and Driver safety. 


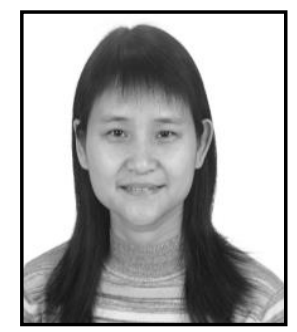

Shu Gao, She is working as a Fulltime Professor at Wuhan University of Technology, Wuhan, China. She has received her Master Degree and $\mathrm{PhD}$ in Computer Science from Wuhan University of Technology, Wuhan, China. Her areas of research are Data mining and Transportation data analysis. 
International Journal of Grid and Distributed Computing Vol. 9, No. 8 (2016) 